Ann. Génét. Sél. anim., r969, 1 (4), 465-468.

\title{
QUELQUES RÉFLEXIONS A L'ISSUE DU SÉMINAIRE SUR L'INTERSEXUALITÉ CHEZ LA GHEVRE SANS CORNES
}

\author{
A. JOS'T et J.-J. LAUVERGNE* \\ Laboratoire de Physiologie Comparée, \\ Faculté des Sciences, 9, quai Saint-Bernard, 75-Paris-5 ${ }^{\mathrm{e}}$ \\ * Station centrale de Génétique animale, \\ Centre national de Recherches zootechniques, 78-Jouy-en-Josas \\ Institut national de la Recherche agronomique
}

Comme le montrent les rapports résumant les travaux, le Séminaire sur l'Intersexualité chez la Chèvre a été fort studieux. Il a aussi donné lieu à des discussions animées. Sans reprendre le détail de ces débats, nous voudrions en dégager les enseignements et ajouter quelques réflexions. L'un d'entre nous résumera quelques aspects génétiques (J.-J. LAUvERGNE) et l'autre considèrera les aspects liés à la différenciation du sexe et à la physiologie du développement (A. Jost).

\section{INTÉRÊTT DU MUTANT ÉTUDIÉ}

Dans l'état actuel des recherches concernant le déterminisme du sexe chez les Mammifères, il est à peine nécessaire de souligner l'intérêt que présente tout gène susceptible de modifier ce processus. Cet intérêt sera d'autant plus grand que le mutant est porté par une espèce sur laquelle il est facile d'expérimenter et qu'il est marqué, c'est-à-dire qu'on peut repérer sa présence par une autre manifestation que l'intersexualité.

Le facteur d'intersexualité avec absence de cornes chez la Chèvre, qui est le thème du Séminaire, répond à ces deux impératifs : l'espèce caprine est déjà une espèce expérimentale couramment utilisée, en physiologie notamment, et le gène d'intersexualité présenté par certaines de ses lignées est associée avec le caractère d'absence de cornes.

\section{IMPORTANCE DU DIAGNOSTIC DU SEXE CHROMOSOMIQUE DES INTERSEXUÉS}

Dès qu'on a affaire à un cas d'intersexualité héréditaire, la première chose à connaître est le sexe chromosomique des anormaux. Dans le cas qui nous occupe, il y a trois types principaux d'anormaux : intersexués (avec de multiples variantes), mâles stériles par hypoplasie et mâles stériles par sténose épididymaire. Les mâles étant en surnombre, on a pensé très vite que les 
intersexués devaient être des femelles génétiques, ce qui a été prouvé. Quant aux mâles stériles, qui sont la plaie des élevages, ce n'est que tout récemment que Soller et al. (1969) ont montré que les uns étaient des femelles génétiques (les hypoplasiques), les autres des mâles génétiques (les sténosiques). Aux examens caryologiques encore peu nombreux de cet auteur sont venus s'ajouter ceux de Basrur et Kanagawa et Corteel et al. Par ailleurs, Weber a montré comment, en Suisse, depuis 10 ans, on détectait par simple examen externe ces deux types de mâles stériles sur les concours de boucs de races sans cornes.

La revue bibliographique que HULOT et BASRUR consacrent à ce problème de détermination du sexe chromosomique montre les aléas de ces recherches à l'époque où les déterminations caryologiques chez la Chèvre étaient fort incertaines, c'est-à-dire avant l'introduction des méthodes récentes sur culture de tissus.

\section{L'ÉVENTAIL DES ACTIONS DU GÈNE MUTANT SELON LES GÉNOTYPES}

Depuis le début des études génétiques dans les années 20 , il s'est écoulé près d'un demisiècle. C'est dire la lenteur avec laquelle on s'est rapproché de la solution. Lauvergne $(a)$ a retracé le progrès des connaissances en la matière et donné une liste des paramètres qu'il est nécessaire d'évaluer pour préciser l'action exacte de ce mutant : il y en a 13 dont un certain nombre sont d'ores et déjà connus, comme la pénétrance des atteintes épididymaires chez les mâles $P p(=0)$ ou de la stérilité des femelles $(=1)$. Pour ceux qu'il est possible d'évaluer au moyen des données de divers auteurs, les estimations ne semblent pas encore définitives; certains seront sans doute impossibles à préciser, par exemple l'expressivité des formes intersexualité, femelle stérile et mâle stérile chez les femelles $P P$ où les frontières entre les phénotypes sont floues. Une des originalités de ce facteur est de provoquer une surprolificité des mâles et des femelles de certains génotypes. Chez les femelles, ce phénomène avait déjà été vu par les Israéliens, chez les mâles le mérite de l'avoir mis en évidence et mesuré revient à RicondeAu. En ce qui concerne l'hérédité du cornage, l'examen révèle que les proportions mendéliennes ne sont guère respectées; Ricordead et Bouillon nous en ont expliqué la raison : une fraction seulement des animaux qui seront cornus sont détectés dans les quelques semaines, sinon les quelques jours, de vie qu'ont la plupart des chevreaux dans leur élevage d'origine avant d'être abattus. Dans ces conditions, il y a toujours un déficit du nombre des cornus. Bien entendu, cela peut masquer d'autres phénomènes, comme une pénétrance incomplète chez certains génotypes.

\section{LES ÉTUDES DE GÉNÉTIQUE DES POPULATIONS ET LES CONSÉQUENCES POUR L'ÉLEVAGE}

Les conceptions que nous avons sur le déterminisme génétique du caractère permettent de bâtir un modèle de génétique des populations qui admet une fréquence d'équilibre du gène $P$ chez les mâles comprise entre 0,521 et 0,661 . Les fréquences observées dans les populations suisses et allemandes se situent dans l'intervalle entre ces valeurs théoriques (LaUvergne, $b$ ). On vérifie ainsi la justesse des hypothèses génétiques. Sur le plan pratique, on constate que le maintien d'une telle situation conduit à éliminer à chaque génération la moitié des femelles et au moins 28 p. 100 des mâles, ce qui est un handicap énorme pour l'amélioration génétique rationnelle de l'espèce.

\section{L'INTERSEXUALITÉ DES CHÈVRES SANS CORNES DU POINT DE VUE DE LA DIFFÉRENCIATION DU SEXE}

Les études génétiques et caryologiques dont on nous a rendu compte au cours du séminaire s'accordent pour indiquer qu'à l'état homozygote le gène autosomique $P$, dominant pour le caractère "absence de cornes " (polled), perturbe l'organogenèse génitale, probablement d'une des partie mâles XY (malformation épididymaire, éventuellement restes mülleriens, d'après les 
faits présentés par Basrur et KaNAGAwa, a) et certainement celle des individus XX, qui devraient devenir des femelles. Ces derniers sujets différencient des glandes génitales de structure testiculaire, en général stériles. Des cellules germinales ont été observées chez un fœtus (Hamerton et al., 1969) et exceptionnellement chez certains adultes. Les causes de l'absence ou de la dégénérescence précoce des cellules germinales dans un testicule de caryotype XX sont encore inconnues. Le fait se rencontre aussi dans d'autres espèces animales et dans l'espèce humaine, et il pose la question de savoir si, chez les Mammifères, une inversion sexuelle complète en gamètes achevés de type opposé à celui attendu du caryotype, est possible.

Les testicules des chèvres $P P$-XX adultes sécrètent des androgènes comme l'ont montré, en particulier, les dosages hormonaux directs dans la veine testiculaire (HARMERTon et al., 1969). Pendant la période foetale, ces testicules masculinisent, éventuellement d'une manière très poussée, l'appareil génital comme le font les testicules des fœtus mâles normaux. Il faudrait étudier avec plus de précision qu'il n'a été possible de le faire jusqu'ici, l'état des dérivés mülleriens, en n'évaluant pas seulement leur volume (qui peut dépendre des hormones circulantes), mais leur état de continuité ou de discontinuité depuis le pavillon de la trompe jusqu'au vagin.

Le problème crucial posé par les chèvres $P P$-XX est celui du déterminisme de la différenciation testiculaire. Malheureusement, nos connaissances sur les processus hormonaux de la différenciation sexuelle de la gonade sont si faibles et si incertaines qu'elles ne peuvent guère servir de point de départ pour l'interprétation du cas particulier des chèvres mottes. Dans le cas général, chez les embryons pourvus d'un caryotype XY la différenciation testiculaire est précoce, alors que chez les embryons $\mathrm{XX}$ la différenciation des gonades appelées à devenir des ovaires est lente et tardive. Tout se passe comme si les gènes de masculinisation provoquaient précocement la différenciation testiculaire dans une ébauche, qui sans ce processus deviendrait un ovaire (cf. Jost, 1969). Les faits présentés par HAMERTon suggèrent qu'un stimulus de masculinisation, se manifestant plus tardivement que chez le mâle normal, peut encore imposer une différenciation testiculaire sur l'ébauche présomptive de l'ovaire. Il reste à expliquer pourquoi, chez les chèvres $P P-\mathrm{XX}$, existe un stimulus de masculinisation gonadique. Des interprétations très ingénieuses ont déjà été proposées, soit au cours de ce Sympoşium, soit antérieurement, mais la question ne peut être encore considérée comme résolue.

\section{EN GUISE DE CONCLUSION}

De toutes les études relatives aux chèvres intersexuées, celles de génétique paraissent les plus avancées, mais elles comportent encore des lacunes.

Les études anatomiques et histologiques réalisées jusqu'ici sont souvent insuffisantes; des examens précis et des comparaisons détaillées avec des animaux témoins doivent être faites; elles profiteront d'une étude embryologique indispensable et devront être faites dans le même esprit d'analyse des structures, de leur développement et de leur fonctionnement; elles rejoindront ainsi les études endocrinologiques déjà amorcées. De telles recherches ne semblent guère réalisables que sur des troupeaux expérimentaux dans lesquels le génotype de tous les sujets sera déterminé, ce que nos connaissances en matière de génétique permettent déjà.

Le type d'intersexualité particulière présenté par les chèvres homozygotes pour le gène $P$ soulève à la fois de passionnants problèmes dans l'étude du déterminisme et de la différenciation du sexe, et des problèmes pratiques pour l'élevage, la sélection et la rentabilité des troupeaux.

Reçu pour publication en avril 1970.

\section{SUMMARY}

SOME THOUGHTS FOLLOWING THE MEETING ON THE INTERSEXUALITY OF HORNIESS GOATS

The anatomical and physiological studies undertaken until this day on intersexuality associated with polledness in the goat are often insufficient. More meticulous examinations and detailed comparisons with groups of control-animals are necessary. These examinations are necessary to introduce the indispensable embryological study and should be undertaken with 
the same spirit of analysing the structures, their development and functionning; they will thus rejoin the endocrinological studies already initiated.

This type of research is only possible in experimental flocks in which the animals' genotype can be determined, which is actually possible with the genetic knowledge we dispose.

The type of particular intersexuality found in goats homozygous for the gene $P$ introduces not only passionate problems on the study of the determinism and the differenciation of sex but also more practical husbandry problems as well as questions of interest to selection and flock rentability.

\section{RÉFÉRENCES BIBLIOGRAPHIQUES}

BASRUR Parvathi K., Kanagawa H., rg69. Anatomic and cytogenetic studies on rg hornless goats with sexual disorders. Ann. Génét. Sél. anim., 1, 349-378.

Corteel J.M., Hulot Françoise, Courot M., Attal J., Phil.rpon A., I969. Examens morphologiques, caryologiques, physiologiques et pathologiques de boucs stériles sans cornes. Ann. Génét. Sél. anim., 1, $34 \mathrm{I}-348$.

Hamerton J.I., Dickson J.M., Pollard C.E., Grieves S.A., Short R.V., I969. Genetic intersexuality in goats. J. Reprod. Fert., Supplem., 7, 25-51.

Hulot Françoise, BasRur Parvathi K., I969. La détermination du sexe chromosomique dans l'étude de l'intersexualité associée à l'absence de cornes chez la chèvre d'origine alpine. Ann. Génét. Sél. anim., 1, 383-390.

Jost A., 1969. Symposium on determination of sex, Lonđon, May 1969. Proc. Roy. Soc. (sous presse).

I.AUVERGne J.J., I969a. Progrès des connaissances génétiques sur l'intersexualité associée à l'absence de cornes chez la chèvre d'origine alpine. Ann. Génét. Sél. anim., 1, 403-412.

LAUVERGNe J.J., I969b. Fréquences à l'équilibre du gène d'origine alpine responsable de l'absence de cornes et de l'intersexualité dans des populations de chèvres. Ann. Génét. Sél. anim., 1, 4I3-422.

Ricordeau G., I969. Surprolificité des génotypes sans cornes dans les races caprines alpines Saanen, alpine chamoisée et poitevine. Ann. Génét. Sél. anim., 1, 39I-395.

Ricordeau G., Boumlon J., r969. Variations de l'âge d'apparition du cornage dans les races caprinel alpines Saanen, alpine chamoisée et poitevine. Ann. Génét. Sél. anim., 1, 397-40r.

Soller M., Padeh B., Wysoki M., Ayalon N., 1969. Cytogenetics of Saanen Goats showing abnormas development of the reproductive tract associated with the dominant gene for polledness. Cytogenetics, 8, $5 x-67$.

WEber W., r969. Ira stérilité des mâles dans les races caprines suisses sans cornes. Ann. Génét. Sél. anim., 1, $379-382$. 